\title{
Buen Vivir, ein Thema für einen BNE-orientierten Geographie- (und Wirtschaftskunde) Unterricht? Analyse von Dokumenten mit unterrichtlichen Lernsettings
}

\author{
* lukas.recknagel@geo.uni-halle.de, Didaktik der Geographie, Institut für Geowissenschaften und Geographie, Martin-Luther- \\ Universität Halle-Wittenberg
}

eingereicht am: 20.03.2018, akzeptiert am: 14.07.2018

\begin{abstract}
Die Diskussionen über Buen Vivir und die damit verbundenen Impulse für die Nachhaltigkeitsdebatte finden zunehmend Eingang in den Diskurs und die Praxis der Bildung für Nachhaltige Entwicklung (BNE). In diesem Artikel werden Dokumente untersucht, die Buen Vivir zum Inhalt von unterrichtlichen Lernsettings machen. Daraus werden Themen, geographische Räume und BNE-Bezüge identifiziert, die auch im Geographieunterricht Anwendung finden.
\end{abstract}

Keywords: Bildung für nachhaltige Entwicklung (BNE), Buen Vivir, Lateinamerika, gutes Leben, Wirtschaftswachstum

\section{Buen Vivir - a topic for ESD oriented Geography and GW-lessons? Analysis of documents on learn- ing settings}

The discussions about Buen Vivir and the associated impulses for the sustainability debate are increasingly included into the discourse and practice of the Education of Sustainable Developement (ESD). In this article documents are examined that topicalize Buen Vivir in schools or teaching. Themes, geographical areas and references to ESD were identified, which can also be used in geography lessons.

Keywords: Education for Sustainable Development (ESD), Buen Vivir, Latin America, good life, economic growth

\section{$1 \quad$ Einleitung}

In den Schulfächern Geographie sowie Geographie und Wirtschaftskunde ${ }^{1}$ nimmt die Bildung für Nachhaltige Entwicklung (BNE) eine wichtige Rolle ein (vgl. DGfG 2017; Hinsch et al. 2014). Daher sollten auch im Unterricht stets aktuell geführte Diskurse um nachhaltige Entwicklung berücksichtigt werden (vgl. Holz \& Stoltenberg 2011), in denen seit einigen Jahren über Buen Vivir diskutiert wird (vgl. KMK \& BMZ 2016; Deutsche UNESCO-Kommission et al. 2016).

Buen Vivir (span. für "gutes Leben“) geht auf indigene Kulturen Lateinamerikas zurück und zielt auf Folgendes ab: „Harmonie und Dialog zwischen allen Menschen sowie zwischen Menschheit und der Natur;

\footnotetext{
Alle Aussagen zum Geographieunterricht oder dem Schulfach Geographie beziehen sich im folgenden Text auch auf den GWUnterricht und das österreichische Schulfach Geographie und Wirtschaftskunde.
}

die Erhaltung der ökologischen Systeme und Kreisläufe; Gerechtigkeit, Solidarität und Würde; Respekt vor Vielfalt; eine Ethik der Verantwortung; und ein harmonisches Leben anstatt linearer Entwicklung" (Kuhn \& Rieckmann 2010: 9). In den Jahren 2008 und 2009 wurde Buen Vivir zu einem Leitbegriff der Verfassung Ecuadors und Boliviens (vgl. López Ayala 2012).

Das Verhältnis von Buen Vivir und Nachhaltigkeit ist kaum erforscht (vgl. Barski 2017), es wird im wissenschaftlichen Diskurs jedoch kontrovers diskutiert: Einerseits gilt Buen Vivir als spezifisch lateinamerikanischer Beitrag zu Nachhaltigkeit, indem der MenschNatur-Dualismus und die expansive Entwicklung kritisiert sowie die Transformation von Wirtschaft und intra- und intergenerationellen Machtbeziehungen gefordert werden (vgl. Vanhulst \& Beling 2014). Andererseits sei Buen Vivir wegen seiner „Rückständigkeit" (Gryl \& Budke 2016: 64) nicht mit dem eurozentrischen Nachhaltigkeitsbegriff kompatibel. 
Zudem könne Nachhaltigkeit nicht auf Konzepte von gutem Leben zurückgreifen, weil für deren Gestaltung objektivierbare Maßstäbe fehlten (vgl. Ekardt 2015).

Vor diesem Hintergrund findet der fachwissenschaftliche Diskurs über Buen Vivir im Kontext einer BNE statt. So könne Buen Vivir im Rahmen einer BNE als Inspiration für neue Wohlstandsmodelle (vgl. Getzin \& Singer-Brodowski 2016) und als transformativer Nachhaltigkeitsansatz thematisiert werden (vgl. Rieckmann 2017). Anhand von Buen Vivir könnten entwicklungstheoretische Denkmuster bei Schülerinnen und Schülern entwickelt (vgl. Fischer et al. 2016), der Wertehorizont erweitert und eurozentrische Denkweisen überwunden werden (vgl. Rieckmann et al. 2014; Rieckmann \& Schank 2016). Da anhand des Buen Vivir Themen angesprochen werden, die auch für den Geographieunterricht relevant sind - u. a. Transformation, Nachhaltigkeit, Entwicklung, Werten, Wohlstand (vgl. Oberrauch \& Keller 2017; Bedehäsing \& Padberg 2017; Ulrich-Riedhammer 2014) - ist die Einschätzung treffend, Buen Vivir könne ein sehr geeigneter Inhalt sein, wenn eine Konkretisierung erfolge (vgl. Rieckmann et al. 2010).

Unerforscht ist bislang, wie und ob Buen Vivir ein Thema für den Geographieunterricht sein kann, ob und wie die oben beschriebenen Bezüge genutzt werden können und wie dabei der fachwissenschaftlichen Kontroversität über den Zusammenhang von Buen Vivir und Nachhaltigkeit Rechnung getragen werden kann.

\section{Forschungsfragen}

Der vorliegende Artikel dient der Diskussion, ob und gegebenenfalls wie Buen Vivir Gegenstand eines BNEorientierten Geographieunterrichts sein könnte. Dazu werden publizierte Dokumente analysiert, in denen Buen Vivir bereits in (unterrichtliche) Lernsettings integriert wird. Dabei sollen folgende Fragen beantwortet werden:

1. Welche Dokumente gibt es, die Buen Vivir zum Gegenstand eines (unterrichtlichen) Bildungskontextes machen?

2. Welche Themen und welche geographischen Räume werden in den Dokumenten im Zusammenhang mit Buen Vivir benannt?

3. Welche Bezüge zu BNE werden in den Unterrichtsdokumenten über Buen Vivir hergestellt?

\section{Methodisches Vorgehen}

Frage 1: Zunächst wurden durch eine Online-Recherche sowie die Sichtung weiterer Literaturquellen 97 Dokumente identifiziert, die Buen Vivir in einem Bildungskontext the- matisieren. Dabei handelt es sich um Präsentationsfolien von Vorträgen, Artikel in Fachzeitschriften, Qualifikationsarbeiten, Unterrichtsmaterialien, Veranstaltungshinweise und Planungsdokumente. Daraus wurden als Untersuchungsmaterial für die weitere Analyse alle Dokumente ausgewählt, die einen klaren Bezug zu schulischer Bildung aufweisen, indem sie Schüler/innen als Adressatinnen und Adressaten oder Schulfächer als Anwendungsgebiet benennen (vgl. Tab. 1).

Frage 2: Entsprechend der Qualitativen Inhaltsanalyse erfolgte zunächst eine Kategorienbildung am Untersuchungsmaterial (vgl. Kuckartz 2016). Im ersten Schritt wurden dabei alle benannten geographischen Räume und alle Themenbereiche ins Kategoriensystem integriert. Anschließend wurden die identifizierten Themen geclustert und so abstrahiert, dass eine Anwendung der Kategorien auf alle Dokumente möglich war. Es folgte die Codierung des gesamten Untersuchungsmaterials mit dem Kategoriensystem. Mithilfe der Analysesoftware MAXQDA konnte daraufhin untersucht werden, mit welchen Themen die geographischen Räume schwerpunktmäßig verbunden wurden. Schwerpunktmäßig bedeutet hier, dass es in den Dokumenten mindestens zehn Verbindungen eines Raumes mit einem Thema gab.

Frage 3: Für die Beurteilung der Dokumente hinsichtlich ihrer Bezüge zur BNE wurden die Kompetenz- und Zielformulierungen des Untersuchungsmaterials analysiert. Grund dafür ist, dass der Erwerb nachhaltigkeitsbezogener Kompetenzen das zentrale Ziel von BNE ist (vgl. Rieckmann 2010). Insofern kann anhand der Kompetenz- und Zielformulierung in den Dokumenten geprüft werden, ob der dazugehörige Bildungsprozess als Beitrag zur Partizipation der Lernenden an einer nachhaltigen Entwicklung konzipiert ist.

Von den unterschiedlichen Kompetenzmodellen für BNE fiel die Wahl auf das Modell „Kernkompetenzen des Lernbereichs Globale Entwicklung", weil es schulspezifisch und für verschiedene Schulfächer anschlussfähig ist (vgl. KMK \& BMZ 2016) und im Fachdiskurs als anerkannt gilt (vgl. Schoof-Wetzig 2014). Anschließend wurde geprüft, ob sich die Kompetenz- oder Zielformulierungen des Untersuchungsmaterials zu einer oder mehreren Kernkompetenzen zuordnen lassen. So kann beispielsweise die Erarbeitung von kritischer Betrachtung von unterschiedlichen Strategien nachhaltigen Wirtschaftes (z. B. 
Buen Vivir) (vgl. Emprechtinger 2015) den Kernkompetenzen „Informationsbeschaffung und -verarbeitung“ sowie „Kritische Reflexion und Stellungnahme“ zugeordnet werden.

Ergänzend wurde das Untersuchungsmaterial daraufhin analysiert, ob BNE jenseits der Kompetenz- und Zielformulierung benannt wird.

\section{$4 \quad$ Ergebnisse}

Die Analyseergebnisse werden in diesem Kapitel gegliedert nach den drei Forschungsfragen dargestellt.

\subsection{Buen Vivir in (unterrichtlichen) Bildungs- kontexten}

Es konnten 14 Dokumente identifiziert werden, die Buen Vivir zum Gegenstand schulischer Bildung machen. Es werden wiederholt die Fächer Geographie, (Gemeinschaftskunde Rechtserziehung) Wirtschaft, Religion, Philosophie/Ethik und Spanisch angesprochen. Als Zielgruppen werden hauptsächlich ältere Schüler/innen benannt, v.a. die Klassenstufen 9-11. Einige Dokumente (z. B. V, XIV) adressieren jedoch auch deutlich jüngere Lernende. Meist wird ein Zeitbedarf von ein bis zwei Unterrichtsstunden

Tab. 1: Dokumente, die Buen Vivir für (unterrichtliche) Bildungskontexte aufbereiten

\begin{tabular}{|c|c|c|c|c|}
\hline Nummer & Autor/in/Hrsg., Jahr, Titel & Unterrichtsfach & Empfohlene Zielgruppe(n) & $\begin{array}{l}\text { Zeitbedarf } \\
\text { (der einzelnen Module) }\end{array}$ \\
\hline I & $\begin{array}{l}\text { Welthaus Bielefeld (Hrsg., 2012): „Buen Vivir - Was heißt } \\
\text { Gutes Leben?". }\end{array}$ & $\begin{array}{l}\text { Praktische Philoso- } \\
\text { phie; Religion }\end{array}$ & Klassen $9 \& 10$ & sechsmal 90 Minuten \\
\hline$\|$ & $\begin{array}{l}\text { Adveniat Referat Bildung/Pastoral (Hrsg., 2013): Buen Vivir. } \\
\text { Das Konzept zum guten Leben aus Bolivien und Ecuador. }\end{array}$ & Religion (?) & $\begin{array}{l}\text { Schüler/innen; } \\
\text { Jugendgruppen }\end{array}$ & keine Angabe (k.A.) \\
\hline III & $\begin{array}{l}\text { Vences, U. (2013): Buen Vivir - Vom „Guten Leben" und } \\
\text { dem Recht darauf. }\end{array}$ & Spanisch & & k. A. \\
\hline \multirow{2}{*}{ IV } & \multirow{2}{*}{$\begin{array}{l}\text { Verband Entwicklungspolitik Niedersachsen e. V. (Hrsg., } \\
\text { 2014): Voll konkret! Methoden zum Globalen Lernen. Das } \\
\text { Begleitheft zum Comic "Voll global!". }\end{array}$} & k.A. & $\begin{array}{l}\text { 10-24 Teilnehmer/innen } \\
\text { ab } 14 \text { Jahren }\end{array}$ & 45 Minuten \\
\hline & & k.A. & $\begin{array}{l}\text { 15-25 Teilnehmer/innen } \\
\text { ab } 14 \text { Jahren }\end{array}$ & 60 Minuten \\
\hline V & $\begin{array}{l}\text { Verein Niedersächsischer Bildungsinitiativen e.V. (Hrsg., } \\
\text { 2014): Die große Globalisierung für kleine Leute. Globa- } \\
\text { les Lernen mit Grundschulkindern. Grundschule. }\end{array}$ & k.A. & Grundschul-Lehrer/innen & k. A. \\
\hline $\mathrm{Vl}$ & $\begin{array}{l}\text { Emprechtinger, M. (2015): Green Economy. Unterrichts- } \\
\text { material zu globaler Ökonomie. }\end{array}$ & Wirtschaft (?) & ab Klasse 9 & 2 Unterrichtseinheiten \\
\hline VII & $\begin{array}{l}\text { NRW-NROs (Hrsg., 2015): Das Globale Lernen in den } \\
\text { Kernlehrplänen von NRW. Anschlussmöglichkeiten - Un- } \\
\text { terrichtsideen - Materialien. }\end{array}$ & $\begin{array}{l}\text { Evangelische } \\
\text { Religion }\end{array}$ & $\begin{array}{l}\text { Sekundarstufe II } \\
\text { (Gymnasium, Gesamtschule) }\end{array}$ & 1-2 Unterrichtseinheiten \\
\hline VIII & $\begin{array}{l}\text { Büter, M. (2016): Buen Vivir - "Gutes Leben" in Bolivien } \\
\text { und im Spanischunterricht. }\end{array}$ & Spanisch & $\begin{array}{l}\text { Sekundarstufe II } \\
\text { (mindestens drei Jahre } \\
\text { Spanisch-Unterricht) }\end{array}$ & k.A. \\
\hline IX & $\begin{array}{l}\text { Gehenzig, M. \& S. Rostock (2016): Die Große Transfor- } \\
\text { mation - Was soll wachsen und was nicht? Wirtschaften } \\
\text { und Leben innerhalb sozialer und ökologischer Grenzen. }\end{array}$ & k.A. & k. A. & k. A. \\
\hline$X$ & $\begin{array}{l}\text { Meyer, C. (Hrsg., 2017): Diercke Erdkunde Einführungs- } \\
\text { phase Niedersachsen. }\end{array}$ & Geographie & Klasse 11 & k. A. \\
\hline$X I$ & $\begin{array}{l}\text { Entwicklungspolitisches Netzwerk Sachsen e.V. (2017): } \\
\text { Buen Vivir. - eine philosophische Textarbeit zu alternativen } \\
\text { Wohlstandsvorstellungen. }\end{array}$ & Ethik & Klasse 10 & $\begin{array}{l}2 \text { Unterrichtseinheiten } \\
\text { bzw. } 80 \text { Minuten }\end{array}$ \\
\hline XII & $\begin{array}{l}\text { Fairbindung e.V.; Konzeptwerk neue Ökonomie e.V. } \\
\text { (2017): Stimmen aus dem Süden. - Ein Gruppenpuzzle zu } \\
\text { wachstumskritischen Positionen. }\end{array}$ & Geographie & Klasse 11 & $\begin{array}{l}2 \text { Unterrichtseinheiten } \\
\text { bzw. 70-90 Minuten }\end{array}$ \\
\hline \multirow[b]{2}{*}{$X I I I$} & \multirow[b]{2}{*}{$\begin{array}{l}\text { Konzeptwerk neue Ökonomie e.V.; Fairbindung e.V. } \\
\text { (2017): Wohlstand und gutes Leben. - Eine Diskussion } \\
\text { über Gemeinsamkeiten und Unterschiede. }\end{array}$} & Ethik & Klasse 10 (Mittelschule) & \multirow[b]{2}{*}{ k.A. } \\
\hline & & $\begin{array}{l}\text { Gemeinschafts- } \\
\text { kunde Rechtserzie- } \\
\text { hung Wirtschaft }\end{array}$ & Klasse 11 (Gymnasium) & \\
\hline XIV & $\begin{array}{l}\text { Kreuzinger, S., D. Mozart \& J. Steigerwald (2017): } \\
\text { BUEN VIVIR. Regenwald und Klimaschutz. Ideen für ein } \\
\text { GUTES LEBEN - hier und anderswo. Aktionen mit Kin- } \\
\text { dern, Jugendlichen und Multiplikator*innen. }\end{array}$ & k.A. & $\begin{array}{l}\text { Kinder ab Grundschulalter; } \\
\text { Jugendliche; Schulklassen; } \\
\text { Multiplikator/innen der BNE; } \\
\text { Eltern; Großeltern; Familien }\end{array}$ & $\begin{array}{l}\text { k. A.; } 20 \text { Minuten } \\
30 \text { Minuten; } 15 \text { Minuten; } \\
30 \text { Minuten; } 20 \text { Minuten; } \\
60 \text { Minuten; k. A.; } \\
\text { mindestens } 20 \text { Minuten }\end{array}$ \\
\hline
\end{tabular}




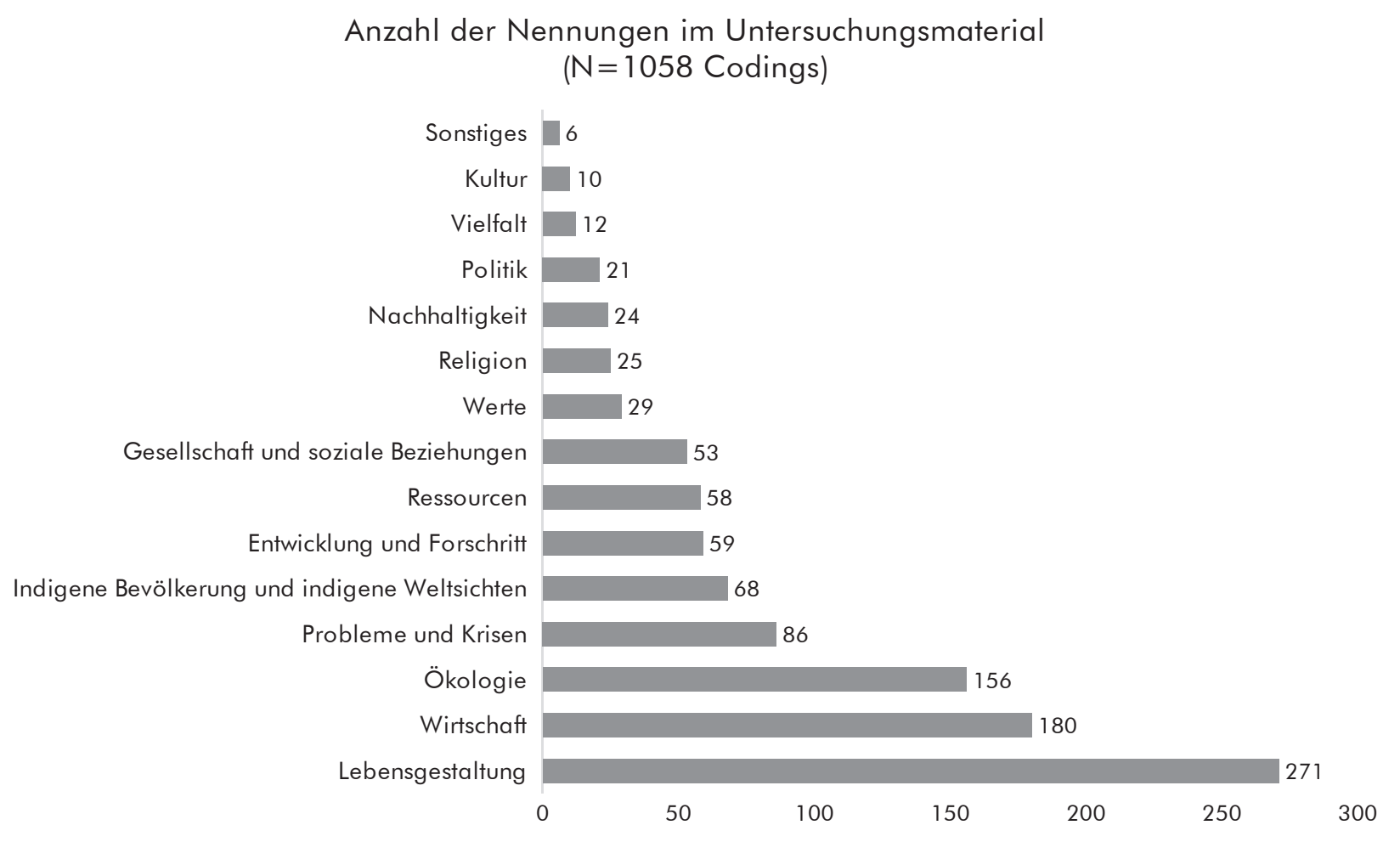

Abb. 1: Themenbereiche im Untersuchungsmaterial; Entwurf: L. Recknagel 2018

vorgesehen; ein Dokument umfasst sechs 90-Minuten-Module, ein anderes neun Einheiten von 15 bis 60 Minuten. Insgesamt fällt auf, dass in jüngerer Vergangenheit ein deutlicher Zuwachs an Dokumenten mit einem Buen-Vivir-Bezug auftritt.

\subsection{Thematische und räumliche Bezüge des Buen Vivir}

In den 14 (in Tab. 1) benannten Dokumenten werden die in Abb. 1 gezeigten Themen dargestellt.

Im nächsten Abschnitt werden kurz die wesentlichen Aussagen zu den einzelnen Themenbereichen zusammengefasst, um die Ausprägung der identifizierten Themen zu verdeutlichen.

Lebensgestaltung: Buen Vivir wird als Anlass verstanden, den eigenen Lebensstil, eigene Vorstellungen zu gutem Leben, die Bedeutung von Lebensmodellen im globalen Kontext usw. zu reflektieren. Dabei wird u.a. darauf Bezug genommen, wie Wohlstand und Glück verstanden werden, was Konsum damit zu tun hat und inwiefern globales Konsumverhalten ein Hindernis für eine gerechte und ökologisch verträgliche Welt ist.

Wirtschaft: Ein Schwerpunkt ist die Abgrenzung des Buen Vivir vom Konzept Wirtschaftswachstum. Zudem wird auf alternative Wirtschaftsformen verwiesen, zu denen Buen Vivir Bezüge hat, v. a. Postwachstum, Gemeingüter und Green Economy.
Des Weiteren werden die wirtschaftliche Situation Ecuadors und Boliviens, globale wirtschaftliche Ungleichheiten, ökologisches Wirtschaften sowie die Funktionsweisen und Wirkungen von Wirtschaftssystemen thematisiert.

Ökologie: Immer wiederkehrendes Element ist das Ziel, Harmonie und Gleichgewicht zwischen Mensch und Natur herzustellen. Begründet liegt dies u.a. in der spirituellen Naturverbundenheit der Indigenen, die sich v. a. im Konzept der Pachamama (Mutter Erde) zeigt. Mensch und Natur seien nicht getrennt zu betrachten, sondern bilden eine Einheit. Die Natur habe Rechte und dürfe nicht ausgebeutet werden. Angesichts von ökologischen Grenzen des Planeten sei eine (weitere) Ausbeutung der Natur unzulässig, es müsse sich viel stärker für Umweltschutz eingesetzt werden. Die Yasuní-ITT-Initiative sei ein Beispiel für die Umsetzung von Buen Vivir in Ecuador.

Probleme und Krisen: Unterschiedliche Krisen und Problemlagen werden aufgegriffen und Buen Vivir als Teil der Lösungssuche bezeichnet. Dabei geht es v.a. um Umweltschäden, Klimawandel, Armut, Disparitäten, Ungerechtigkeiten und Wirtschaftskrise.

Indigene Bevölkerung und indigene Weltsichten: Es wird auf den Einfluss der indigenen Weltsichten auf das Buen Vivir verwiesen, wonach eine Harmonie von allem erreicht werden solle. Weiterhin geht 
es um politische Partizipation Indigener. Häufig wird der Kontrast der vielfältigen indigenen Lebensweisen mit ,unseren' Lebensweisen geschildert.

Entwicklung und Fortschritt: Wichtiges Element des Buen Vivirsei die Abgrenzung vom, westlichen'Entwicklungs- und Fortschrittsbegriff, der meist mit Modernisierung und Wirtschaftswachstum in Verbindung gesetzt wird. Basierend auf den indigenen Einflüssen gehe es Buen Vivirum sozialen Fortschritt und neue Entwicklungswege. Weiterhin kommen kritische Betrachtungen zu Zielen, Mitteln und Folgen globaler Entwicklungspolitik zur Sprache.

Ressourcen: Buen Vivir stehe für mehr Ressourcenschutz. Dies wird wiederholt in den Kontext globalen Ressourcenverbrauchs (v. a. Erdöl, Erdgas und Metalle) gerückt.

Gesellschaft und soziale Beziehungen: Schwerpunkt bei gesellschaftlichen Belangen seien Fragen des $\mathrm{Zu}-$ sammenhalts. Es geht häufig um Gerechtigkeit und gutes Leben in der Gemeinschaft und es wird diskutiert, welche Gesellschaft angestrebt werden sollte.

Werte: Mit der Thematisierung von Buen Vivir wird die Auseinandersetzung mit eigenen Werten (unter besonderer Berücksichtigung der globalen Situation, der Mensch-Natur-Beziehungen und hinsichtlich der Konsequenzen für die eigene Lebensweise) angeregt.

Religion: Buen Vivir habe spirituelle Implikationen und wird in den Dokumenten hinsichtlich religiöser Anschlussmöglichkeiten ans Christentum diskutiert.

Nachhaltigkeit: Bildung sei ein wichtiger Bestandteil einer nachhaltigen Entwicklung. Thematisch geht es um nachhaltige Wirtschaft, nachhaltigen Umgang mit der Umwelt, nachhaltige Lebensstile, die Sustainable Development Goals, Nachhaltigkeitsstrategien und um Zielkonflikte der Nachhaltigkeit.

Politik: Buen Vivir wird einerseits mit den politischen Veränderungen Lateinamerikas in Verbindung gebracht, andererseits werden Anfragen an politische Zielsetzungen, Prozesse und Entscheidungen im weltweiten Kontext formuliert.

Vielfalt: Neben der mehrfach benannten soziokulturellen Vielfalt der Erde werden am Beispiel des Yasuní-Nationalparks Aspekte der Biodiversität dargestellt.

Kultur: Buen Vivir hat viel mit kulturellen Aspekten und kultureller Anerkennung indigener Lebensweise zu tun. Kultur sei dabei nicht von Politik, Wirtschaft und Sozialem zu trennen.

Sonstiges: Vereinzelt werden Große Transformation und Globalisierung erwähnt.
Folgende Räume werden in den Dokumenten im Kontext von Buen Vivir angesprochen:

Tab. 2: Im Untersuchungsmaterial benannte geographische Räume ( $N=307$ Codings)

\begin{tabular}{|c|c|c|c|}
\hline $\begin{array}{l}\text { Maßstabs- } \\
\text { ebene }\end{array}$ & $\begin{array}{l}\text { Geographi- } \\
\text { scher Raum }\end{array}$ & $\begin{array}{l}\text { Nen- } \\
\text { nungen }\end{array}$ & $\begin{array}{l}\text { Themen-Schwerpunkte } \\
\text { (mindestens zehn Schnitt- } \\
\text { stellen zu den Themen auf } \\
\text { dieser Maßstabsebene) }\end{array}$ \\
\hline Globale Ebene & Erde & 64 & $\begin{array}{l}\text { Probleme, Krisen; } \\
\text { Ökologie; Wirtschaft }\end{array}$ \\
\hline \multirow[t]{2}{*}{ Kontinent } & $\begin{array}{l}\text { Süd- bzw. } \\
\text { Lateinamerika }\end{array}$ & 35 & $\begin{array}{l}\text { Indigene und indigene } \\
\text { Weltsicht; Lebensgestaltung; } \\
\text { Wirtschaft }\end{array}$ \\
\hline & Europa & 13 & \\
\hline \multirow[t]{7}{*}{ Nationalstaat } & Ecuador & 59 & $\begin{array}{l}\text { Indigene und indigene } \\
\text { Weltsicht; Lebensgestaltung; } \\
\text { Ökologie; Ressourcen; } \\
\text { Wirtschaft }\end{array}$ \\
\hline & Bolivien & 40 & Lebensgestaltung; Wirtschaft \\
\hline & Peru & 4 & \\
\hline & Deutschland & 22 & Lebensgestaltung \\
\hline & USA & 8 & \\
\hline & weitere Staaten & 5 & \\
\hline & $\begin{array}{l}\text { ohne konkrete } \\
\text { Benennung }\end{array}$ & 9 & \\
\hline Lokale Ebene & & 16 & Ökologie \\
\hline \multirow[t]{3}{*}{ "Abstrakt" } & $\begin{array}{l}\text { Globaler } \\
\text { Süden }\end{array}$ & 11 & \\
\hline & $\begin{array}{l}\text { Globaler } \\
\text { Norden }\end{array}$ & 1 & \\
\hline & "Der Westen" & 20 & Lebensgestaltung; Wirtschaft \\
\hline
\end{tabular}

Es werden unterschiedliche Maßstabsebenen benannt. Auf Ebene der Kontinente werden in den Dokumenten explizit nur Süd- bzw. Lateinamerika sowie Europa benannt. Die Ebene der Nationalstaaten wird konkretisiert durch die Länder Ecuador, Bolivien, Peru, Deutschland und USA. Weitere Staaten treten als Einzelfälle auf. Zudem wird wiederholt von „Entwicklungsländern“, „Industrienationen“ und „anderen Ländern" gesprochen, die nicht weiter expliziert werden. Auf lokaler Ebene wird meist das Beispiel des Yasuní-Nationalparks thematisiert. Zudem finden sich abstrakte Begriffe von Raumkonstruktionen: Globaler Süden, Globaler Norden sowie der Westen (im Sinne von ,westliche Lebensweise'). Die letzte Spalte in Tab. 2 zeigt, welche Themen auf der jeweiligen Maßstabsebene besonders häufig benannt werden.

\subsection{BNE-Orientierung der Buen-Vivir-Bildungs- einheiten}

Insgesamt wurden 67 Kompetenz- oder Zielformulierungen identifiziert. Sie entstammen den Dokumenten I, II, IV, VI, VII, VIII, XI, XII, XIII, XIV (Nummerierung aus Tab. 1) und wurden folgenden Kernkompetenzen des Lernbereichs Globale Entwicklung zugeordnet: 
Tab. 3: Zuordnung der Kompetenz- und Zielformulierung aus dem Untersuchungsmaterial zu den Kompetenzbereichen des Lernbereichs Globale Entwicklung

\begin{tabular}{|c|c|c|c|}
\hline \multicolumn{3}{|r|}{ Kompetenzbereich } & $\begin{array}{l}\text { Zuord- } \\
\text { nungen }\end{array}$ \\
\hline \multirow{4}{*}{ 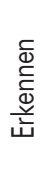 } & 1 & Informationsbeschaffung und -verarbeitung & 11 \\
\hline & 2 & Erkennen von Vielfalt & 6 \\
\hline & 3 & Analyse des globalen Wandels & 1 \\
\hline & 4 & Unterscheidung von Handlungsebenen & 4 \\
\hline \multirow{3}{*}{ 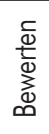 } & 5 & Perspektivenwechsel und Empathie & 25 \\
\hline & 6 & Kritische Reflexion und Stellungnahme & 8 \\
\hline & 7 & Beurteilen von Entwicklungsmaßnahmen & 6 \\
\hline \multirow{4}{*}{$\begin{array}{l}\frac{c}{0} \\
\frac{10}{0} \\
\text { 호 }\end{array}$} & 8 & Solidarität und Mitverantwortung & 4 \\
\hline & 9 & Verständigung und Konfliktlösung & 1 \\
\hline & 10 & Handlungsfähigkeit im globalen Wandel & 2 \\
\hline & 11 & Partizipation und Mitgestaltung & 8 \\
\hline
\end{tabular}

Die Kompetenzbereiche mit den meisten Zuordnungen seien im Folgenden näher beschrieben:

Perspektivenwechsel und Empathie: Hierbei wird Buen Vivir meist als Anlass genommen, die eigene Perspektive auf Werte, die eigene Lebensweise, gutes Leben, Wohlstand, Lebensstile oder Glück wahrzunehmen und (kritisch) zu reflektieren. Darüber hinaus geht es um Entwicklung von Empathie für die Lebensgestaltung anderer Menschen (v.a. Indigener) sowie das Einüben von Perspektivenwechsel (bspw. anhand der Sicht auf Entwicklung von Expertinnen und Experten aus dem Globalen Süden).

Informationsbeschaffung und -verarbeitung: Die Lernenden informieren sich zu verschiedenen Inhalten (bspw. Strategien nachhaltiger Entwicklung, Globalisierung, Bedeutung des Regenwaldes) und erkennen die eigenen Vorstellungen zu Glück, einer gerechten, umweltverträglichen Lebensweise und zu gutem Leben.

Partizipation und Mitgestaltung: Alle acht Zuordnungen beziehen sich auf das Dokument XIV. Dabei geht es um die Entwicklung bzw. das Erkennen von Handlungsmöglichkeiten bezüglich des Regenwald- und Ressourcenschutzes, um die Stärkung von Kreativität, Entscheidungsfindung, umweltsensiblen Handlungen und Motivation sowie das Üben bestimmter Handlungen (teilen, tauschen, verschenken).

Kritische Reflexion und Stellungnahme: Die Lernenden sollen sich zu nachhaltiger Entwicklung, Buen Vivir, dem eigenen Konsum und Lebensstil positionieren und ein Bewusstsein für die globale Perspektive nachhaltiger Entwicklung oder die Bedeutung des Regenwaldes bilden.

Auch jenseits der Passung von Kompetenz- und Zielformulierung mit den Kernkompetenzen des
Lernbereichs Globale Entwicklung wird deutlich, dass die Dokumente im Zusammenhang bzw. erweiterten Kontext mit BNE stehen: durch Verweise auf Gestaltungskompetenz (IX, XIV), „Methoden der BNE“ (XIV), die anzusprechenden Adressatinnen und Adressaten (XIV), durch Erwähnung von BNE (VI, X) oder die Bezugnahme zu Globalem Lernen (IV, VII, VIII). Nur zwei Dokumente lassen keine Hinweise auf BNE oder Globales Lernen erkennen (III, V).

\section{Diskussion der Ergebnisse für einen BNE-orientierten Geographieunterricht}

Zunächst kann festgehalten werden, dass es für den Geographieunterricht bereits Ideen gibt, Buen Vivir zu thematisieren (X, XII) und auch Möglichkeiten für fachübergreifenden Unterricht bestehen (v. a. mit Ethik/Philosophie, Religion, Wirtschaft, Spanisch). Die Dokumente konzentrieren sich schwerpunktmäßig auf ältere Schüler/innen, eine Implementation von Buen Vivir scheint in höheren Jahrgängen naheliegender zu sein als bei jüngeren Schülerinnen und Schülern. So ist es auch angesichts der inhaltlichen Breite und Komplexität sinnvoll, auf Vorwissen zu Themen wie Nachhaltigkeit zurückgreifen zu können.

Inhaltlich werden in den Dokumenten unterschiedliche Themen aufgegriffen, die auch für die Geographie relevant sind. Mit Ökologie, Wirtschaft, Politik, Kultur, Gesellschaft und sozialen Beziehungen werden wesentliche Nachhaltigkeitsdimensionen angesprochen (vgl. KMK \& BMZ 2016). Davon, dass diese Themen auch für den Geographieunterricht relevant sind, zeugen sowohl die Hauptbasiskonzepte als auch das Leitbild Nachhaltigkeit (vgl. DGfG 2017). Gerade auf die Themen Wirtschaft und Ökologie bietet Buen Vivir Perspektiven Lateinamerikas; hierbei kann auch das Thema ,Indigene Bevölkerung und indigene Weltsichten', verbunden mit einem passenden Raumbeispiel, Inhalte für den Geographieunterricht bieten.

Buen Vivir bietet eine Weltsicht, in der Mensch und Natur nicht zu trennen sind und eine Einheit bilden. Diese Position kann als Spiegel der eigenen Beziehung zur Natur dienen sowie als Bewertungsmaßstab für Nachhaltigkeitsstrategien. Bei ökonomischen Fragen liegt ein Schwerpunkt des Buen Vivir auf der Dekonstruktion der scheinbaren Notwendigkeit von Wirtschaftswachstum; so bietet es auch Zugang zu alternativen Wirtschaftsweisen. Die Bedeutung des Hinterfragens neoliberaler Diskurse sowie von (ebenso im Buen Vivir-Kontext explizierter) Entwicklungskritik sowie die Kategorie ,Eigenwert der $\mathrm{Na}$ tur' haben vor dem Hintergrund des transformativen Anspruchs von BNE entscheidende Bedeutung (vgl. Singer-Brodowski 2016). 
Die Yasuní-ITT-Initiative ist eine Möglichkeit, Buen Vivir in seiner politischen Umsetzung zu betrachten. Eine Besonderheit ist die Verbindung von Klimaschutz, Schutz von Biodiversität und indigenen Völkern sowie Entwicklungspolitik im globalen Maßstab. Hier sei angemerkt, dass die Grundidee dieses Projekts in den meisten Darstellungen nur positiv bewertet wird. Das Erkenntnispotenzial wird durch kritische Stimmen an der Initiative erhöht, wie das Argument, dass eine Nicht-Förderung von Ressourcen aufgrund von Ausgleichszahlungen einer Explorationssubvention gleichkomme. Die Yasuní-ITT-Initiative steht auch exemplarisch für den Zielkonflikt zwischen Wirtschaft und Umwelt (vgl. KMK \& BMZ 2016).

Ein Großteil der anderen identifizierten Themen hat im Geographieunterricht ebenso Berechtigung; so greifen die Bildungsstandards Entwicklung, Probleme, Ressourcen, Vielfalt und Werte auf; einzig Religion und Lebensgestaltung werden in den Bildungsstandards nicht vertieft. Gerade die Lebensgestaltung ist jedoch ein wesentlicher Schwerpunkt in den Dokumenten: Dieses Thema hat nicht nur die meisten Zuordnungen, es spielt auch bei den benannten geographischen Räumen eine zentrale Rolle und ist insofern interessant für den Geographieunterricht, da gerade die Auseinandersetzung mit eigenen Vorstellungen von Lebensqualität hohe Bedeutung für eine BNE hat. Wichtig sind dabei einerseits der Perspektivenwechsel, der durch die Auseinandersetzung mit lateinamerikanischen Positionen auf gutes Leben, Wohlstand und Lebensqualität angedeutet werden kann, sowie die Realisierung der sozial-kulturellen Konstruktion dieser Vorstellungen. Eine Auseinandersetzung mit Lebensgestaltungen aus globaler Perspektive, gerade einer fremden Kultur, kann hohes Selbsterkenntnispotenzial bieten (vgl. Oberrauch \& Keller 2017). Die hier angedeuteten indigenen Kulturen Lateinamerikas können dabei auch Thema eines Geographieunterrichts sein, der interkulturelles Lernen fördert.

Dass Buen Vivir die Reflexion fremder und eigener Perspektiven auf die Lebensgestaltung anstößt, zeigt sich auch bei den in den Dokumenten auftretenden Kompetenzbereichen: Es scheinen sich im Zusammenhang mit Buen Vivir besonders ,Perspektivenwechsel und Empathie' sowie, Kritische Reflexion und Stellungnahme' anzubieten. Dabei geht es darum, anhand der Fremderfahrung, wie beim Buen Vivir gutes Leben, Wohlstand oder Lebensqualität dargestellt wird, die eigene Position besser wahrzunehmen sowie kritisch Stellung beziehen zu können.

Diese Ausführungen deuten nur an, welche Zugänge zu Buen Vivir für einen BNE-orientierten Geographieunterricht denkbar sind, die folgende Struktur haben könnten:
Tab. 4: Beispielsequenz zu Buen Vivir und nachhaltiger Entwicklung im Geographieunterricht

\begin{tabular}{|c|c|c|c|}
\hline $\begin{array}{l}\text { Ab- } \\
\text { schnitt }\end{array}$ & $\begin{array}{l}\text { BNE-Kompetenz- } \\
\text { bereich }\end{array}$ & Inhalt & $\begin{array}{l}\text { Material- } \\
\text { empfeh- } \\
\text { lung }\end{array}$ \\
\hline 1 & $\begin{array}{l}\text { Perspektivenwechsel } \\
\text { und Empathie }\end{array}$ & $\begin{array}{l}\text { Indigene Lebenswelten in } \\
\text { Lateinamerika }\end{array}$ & $\begin{array}{l}\text { I, S. } 11 \mathrm{f} . \\
\text { XII, S. } 15 \mathrm{f} .\end{array}$ \\
\hline 2 & $\begin{array}{l}\text { Informationsbe- } \\
\text { schaffung und } \\
\text {-verarbeitung }\end{array}$ & $\begin{array}{l}\text { Harmonie von Natur und } \\
\text { Mensch - nur eine Utopie? }\end{array}$ & $\begin{array}{l}\text { I, S. 11, } \\
13 \mathrm{f} ., 28 \\
\text { XII, S. } 15 \mathrm{f} .\end{array}$ \\
\hline 3 & $\begin{array}{l}\text { Beurteilen von } \\
\text { Entwicklungsmaß- } \\
\text { nahmen } \\
\end{array}$ & $\begin{array}{l}\text { Die Yasuní-ITT-Initiative - ein } \\
\text { sinnvoller Beitrag zu einer } \\
\text { nachhaltigen Entwicklung? }\end{array}$ & $x$ \\
\hline 4 & $\begin{array}{l}\text { Unterscheidung von } \\
\text { Handlungsebenen }\end{array}$ & $\begin{array}{l}\text { Die Position des Buen Vivir } \\
\text { zu Wirtschaftswachstum } \\
\text { - eine global relevante } \\
\text { Einschätzung? }\end{array}$ & $\begin{array}{l}\text { I, S. } 27 \\
\text { XII, S. } 15 f .\end{array}$ \\
\hline 5 & $\begin{array}{l}\text { Analyse des globa- } \\
\text { len Wandels }\end{array}$ & $\begin{array}{l}\text { Globales Wohlstandsstreben } \\
\text { als Triebfeder von Wirt- } \\
\text { schaftswachstum? }\end{array}$ & $\begin{array}{l}\text { I, S. } 29 \\
\text { IX } \\
\text { XIII, S. } 4\end{array}$ \\
\hline 6 & $\begin{array}{l}\text { Kritische Reflexion } \\
\text { und Stellungnahme }\end{array}$ & $\begin{array}{l}\text { Die Vereinbarkeit persönli- } \\
\text { cher Vorstellungen von gutem } \\
\text { Leben mit Erfüllung der SDGs }\end{array}$ & $\begin{array}{l}\text { I, S. } 1 \mathrm{f} . \\
\text { IV, S. } 28\end{array}$ \\
\hline 7 & $\begin{array}{l}\text { Solidarität und } \\
\text { Mitverantwortung }\end{array}$ & $\begin{array}{l}\text { Verortung der eigenen } \\
\text { Position im Kontext globaler } \\
\text { Nachhaltigkeitsbestrebungen }\end{array}$ & $\begin{array}{l}\text { II, S. } 22 \\
\text { IX } \\
\text { XII, S. } 15 f .\end{array}$ \\
\hline
\end{tabular}

\section{Fazit}

Mithilfe der Dokumentenanalyse konnten einige Potenziale des Buen Vivir, die im Fachdiskurs geäußert wurden, mit Inhalten von BNE-orientierten Bildungsprozessen verbunden werden, z. B. die Auseinandersetzung mit Wohlstandsmodellen (vgl. Getzin \& Singer-Brodowski 2016), Werten (vgl. Rieckmann \& Schank 2016) und Entwicklungspolitik (vgl. Fischer et al. 2016). Darüber hinaus wurde festgestellt, dass weitere Themen eine Rolle spielen können (Ökologie, Indigene \& indigene Weltsicht, Wertesysteme), und es konnten Anhaltspunkte gesammelt werden, zu welchen BNE-relevanten Kompetenzbereichen Buen Vivir als Inhalt passt (v.a. ,Perspektivenwechsel und Empathie'). Die Zuordnung der Zielkompetenzen des Lernbereichs Globale Entwicklung zu den Kompetenzen und Zielen in den Dokumenten ist aufgrund der unterschiedlichen Formulierungsebenen kompliziert.

Inwiefern die Potenziale des Buen Vivir im BNEKontext unabhängig von der hier dargestellten Verbindung mit BNE auch gewünschte Lerneffekte erzielen, müssen empirische Wirksamkeitsstudien zeigen. Darüber hinaus fand mit der hier gewählten Vorgehensweise nur Berücksichtigung, was bereits zum Buen Vivir im Bildungskontext ausgearbeitet wurde. Für die analysierten Materialien wurde schon eine Reduktion und Selektion von Inhalten des Buen Vivir vorgenommen. Daher wäre anhand anderer Materialien, die Buen Vivir ausführlich darstellen, zu prüfen, ob 
sie weitere Ansätze für eine BNE bieten als die, die in den Dokumenten angesprochen werden. Insofern sind auch einige der Ergebnisse der vorliegenden Analyse in ihrer Aussagekraft einzuschränken. Es ist zwar auffällig, dass Buen Vivir vor allem anhand ,klassischer Nachhaltigkeitsthemen' konkretisiert wird, was den Schluss zulässt, dass Buen Vivir eine besondere Nähe zu Nachhaltigkeit hat; andererseits zeigt sich, dass Buen Vivir in den Dokumenten meist in einen BNE-Kontext gerückt wurde und somit eine Schwerpunktsetzung mit Blick auf Nachhaltigkeitsthemen ohnehin zu erwarten ist.

Bietet sich also die Beschäftigung mit Buen Vivir in einem BNE-orientierten Geographieunterricht an? Die Frage kann insofern positiv beantwortet werden, dass sich unterschiedliche Themen eignen (v.a. Lebensgestaltung, Ökologie und Wirtschaft), dass sich regionale Bezüge zu Lateinamerika (v.a. Ecuador und Bolivien) und Nachhaltigkeit finden lassen und dass eine Verbindung des Inhalts Buen Vivir mit Kompetenzen einer BNE möglich ist.

Völlig offen ist, welche Lerneffekte bei den Lernenden eintreten. Hinweise und ggf. Motivation dafür liefert die Äußerung einer Schülerin, die sich im Laufe ihrer Schullaufbahn in einer schulinternen Arbeitsgruppe mit Buen Vivir befasste:

„[...] Durch das Buen Vivir erkennen wir nun die Fehler im System [...] Buen Vivir schafft uns einen Weg raus aus dem stressigen, konkurrenzgeprägten Schulalltag hin zur Weltorientierung. Das Konzept löst den Schleier vor unseren Augen und lässt uns erblicken, dass Weltorientierung unausweichlich ist, allerdings innerhalb des Schulunterrichts eher selten geboten wird. Wir haben nun die Möglichkeit, uns mit Problemen auseinanderzusetzen, die uns wirklich betreffen und nachhaltig beeinflussen. Buen Vivir weist uns den Weg zu relevanten und aktuellen Themen und zu einer harmonischen und nachhaltigen Lebensweise. Es ermöglicht uns einen Umgang mit der Frage, warum wir hier sitzen, obwohl es so viel zu tun gibt und fordert uns auf, Lösungen zu finden. So fanden wir in der Weltanschaunng, die den Weltverbund alles Lebendigen ins Zentrum stellt, eine immense Kraftquelle. Wir lernen Neues zu wagen und erkennen, was uns selbst wirklich wichtig ist und wichtig sein sollte. Deswegen wollen wir uns bemühen, Buen Vivir als wesentlichen Bestandteil in den Schulunterricht zu integrieren"(Freitag 2017: 28).

\section{$7 \quad$ Literatur}

Adveniat Referat Bildung/Pastoral (Hrsg., 2013): Buen Vivir. Das Konzept zum guten Leben aus Bolivien und Ecuador. https://www.adveniat.de/fileadmin/user_up-
load/engagieren/Schule_und_Kindergarten/BuenVivir. pdf (25.6.2018).

Barski, C. (2017): Die Bedeutung des Buen Vivir für die Transformation zu einer nachhaltigen Gesellschaft aus Sicht diskursintegrierter zivilgesellschaftlicher Akteure der sozial-ökologischen Bewegung. https://geo.unigreifswald.de/fileadmin/uni-greifswald/fakultaet/mnf/ geowissenschaften/Arbeitsbereiche_Geographie/Nachhaltigkeitswissenschaften/Seite_MSc.Arbeiten/MA_C. Barski.pdf (21.6.2018).

Bedehäsing, J. \& S. Padberg (2017): Globale Krise, Große Transformation, Chage Agents: Heiße Eisen für die Geographiedidaktik? In: GW-Unterricht 146, 19-31. http://www. austriaca.at/0xclaa500e\%200x0036937a.pdf(25.6.2018).

Büter, M. (2016): Buen Vivir - „Gutes Leben“ in Bolivien und im Spanischunterricht. In: Hispanorama 154, 66-75.

Deutsche UNESCO-Kommission, Schweizerische UNESCO-Kommission \& Österreichische UNESCOKommission (2016): Bildung überdenken. ein globales Gemeingut? http://unesdoc.unesco.org/ images/0024/002464/246481ger.pdf (25.6.2018).

DGfG - Deutsche Gesellschaft für Geographie (2017): Bildungsstandards im Fach Geographie für den Mittleren Schulabschluss - mit Aufgabenbeispielen. Bonn: Selbstverlag D.

Ekardt, F. (2015): Theorie der Nachhaltigkeit. Rechtliche, ethische und politische Zugänge - am Beispiel von Klimawandel, Ressourcenknappheit und Welthandel. Baden-Baden: Nomos.

Emprechtinger, M. (2015): Green Economy. Unterrichtsmaterial zu globaler Ökonomie. http://www.baobab.at/ images/doku/ak_green_economy_low.pdf (25.6.2018).

Entwicklungspolitisches Netzwerk Sachsen e.V. (2017): Buen Vivir - eine philosophische Textarbeit zu alternativen Wohlstandsvorstellungen. https://bne-sachsen.de/sites/default/files/materialien/BuenVivir.pdf (25.6.2018).

Fairbindung e.V. \& Konzeptwerk neue Ökonomie e.V. (2017): „Stimmen aus dem Süden“- Ein Gruppenpuzzle $\mathrm{zu}$ wachstumskritischen Positionen. Entwicklungspolitisches Netzwerk Sachsen e.V. https://bne-sachsen.de/ sites/default/files/materialien/StimmenAusDemSueden. pdf (25.6.2018).

Fischer, S., F. Fischer, M. Kleinschmidt \& D. Lange (2016): Globalisierung und Politische Bildung. Eine didaktische Untersuchung zur Wahrnehmung und Bewertung der Globalisierung. Wiesbaden: Springer VS (Bürgerbewusstsein, Schriften zur Politischen Kultur und Politischen Bildung).

Freitag, K. (2017): „Warum sitze ich hier, wenn es doch so viel zu tun gibt?". Buen Vivir - ein neuer Blick auf die Welt und den Schulalltag. In: PÄDAGOGIK 7-8, 26-31.

Gehenzig, M. \& S. Rostock (2016): Die Große Transformation - Was soll wachsen und was nicht? Wirtschaften und Leben innerhalb sozialer und ökologischer Grenzen. Bildung für nachhaltige Entwicklung Arbeitsblätter zum Thema Wachstum. Germanwatch. https://germanwatch. org/de/download/17717.pdf (25.6.2018).

Getzin, S. \& M. Singer-Brodowski (2016): Transformatives Lernen in einer Degrowth-Gesellschaft. In: SOCIENCE $1,33-46$.

Gryl, I. \& A. Budke (2016): Bildung für nachhaltige Entwicklung - Zwischen Utopie und Leerformel? Potenziale für die Politische Bildung im Geographieunterricht. In: 
Budke, A. \& M. Kuckuck (Hrsg.): Politische Bildung im Geographieunterricht. Stuttgart: Franz Steiner, 57-75.

Hinsch, S., H. Pichler, T. Jekel, L. Keller \& F. Baier (2014): Semestrierter Lehrplan AHS, Sekundarstufe II. Ergebnis der ministeriellen Arbeitsgruppe. In: GW-Unterricht 136, 51-61. http://www.gw-unterricht.at/images/pdf/gwu_136_51_61_ hinsch_pichler_jekel_keller_baier.pdf

(21.6.2018).

Holz, V. \& U. Stoltenberg (2011): Herausforderungen einer Bildung für nachhaltige Entwicklung im Spannungsfeld von Alltagstauglichkeit, strukturellen Bedingungen und dem Transfer konzeptueller Komplexität. In: Banse, G., R. Janikowski \& A. Kiepas (Hrsg.): Nachhaltige Entwicklung - transnational. Sichten und Erfahrungen aus Mitteleuropa. Berlin: Edition Sigma, 179-195.

KMK \& BMZ - Kultusministerkonferenz \& Bundesministerium für wirtschaftliche Zusammenarbeit und Entwicklung (Hrsg., 2016): Orientierungsrahmen für den Lernbereich Globale Entwicklung im Rahmen einer Bildung für nachhaltige Entwicklung. Ein Beitrag zum Weltaktionsprogram „Bildung für nachhaltige Entwicklung“. Bonn: Cornelsen.

Konzeptwerk neue Ökonomie e.V. \& Fairbindung e.V. (2017): Wohlstand und gutes Leben - Eine Diskussion über Gemeinsamkeiten und Unterschiede. Entwicklungspolitisches Netzwerk Sachsen e. V. https://bne-sachsen.de/sites/default/files/materialien/UB01_WohlstandUGutesLebenfinal.pdf (25.6.2018).

Kreuzinger, S., D. Mozart \& J. Steigerwald (2017): BUEN VIVIR. Regenwald und Klimaschutz. Ideen für ein GUTES LEBEN - hier und anderswo. Aktionen mit Kindern, Jugendlichen und Multiplikator*innen. http://www.oekoprojekt-mobilspiel.de/download. php? file=download/download_401/BuenVivir_ A5_28Seiten_Webversion.pdf (25.6.2018).

Kuckartz, U. (2016): Qualitative Inhaltsanalyse. Methoden, Praxis, Computerunterstützung. Weinheim: Beltz Juventa.

Kuhn, K. \& M. Rieckmann (2010): Der lateinamerikanische Nachhaltigkeitsdiskurs - von der Kapitalismuskritik zum „Guten Leben“. http://www.kooperation-international.de/fileadmin/public/downloads/itb/ info_10_07_13_SAG.pdf (25.6.2018).

López Ayala, T. (2012): Das Buen Vivir als alternatives Entwicklungskonzept. https://www.researchgate.net/ publication/317956230_Das_Buen_Vivir_als_alternatives_Entwicklungskonzept (25.6.2018).

Meyer, C. (Hrsg.) (2017): Diercke Erdkunde Einführungsphase Niedersachsen. Braunschweig: Westermann.

NRW-NROs (Hrsg.) (2015): Das Globale Lernen in den Kernlehrplänen von NRW. Anschlussmöglichkeiten - Unterrichtsideen - Materialien. http://www.globales-lernenschule-nrw.de/uploads/media/Gy-Ge-ev-Religion-Sek-IIQ-Phase-GK_LK-Buen-vivir-das_gute-Leben.pdf(25.6.2018).

Oberrauch, A. \& L. Keller (2017): Vorstellungen von Jugendlichen zur Lebensqualität zwischen Materialismus und Umweltoreintierung. Eine empirische Untersuchung im Kontext (einer Bildung für) nachhaltige(r) Entwicklung. In: Zeitschrift für Geographiedidaktik 1, 3-32.

Rieckmann, M. (2010): Die globale Perspektive der Bildung für eine nachhaltige Entwicklung. Eine europäischlateinamerikanische Studie zu Schlüsselkompetenzen für Denken und Handeln in der Weltgesellschaft. Berlin: Berliner Wissenschaftsverlag.
Rieckmann, M., M. Adomßent \& P. Aguirre (2010): Das internationale Seminar „Bildung für nachhaltige Entwicklung " in Ecuador - ein Ort Globalen Lernens? In: Zeitschrift für internationale Bildungsforschung und Entwicklungspädagogik 33, 21-26.

Rieckmann, M., D. Fischer \& S. Richter (2014): Nachhaltige Ernährung im Wertediskurs - Beiträge einer Hochschulbildung für nachhaltige Entwicklung. In: Vorbohle, K., J. Quandt \& C. Schank (Hrsg.): Perspektive Nahrungsmittelethik. Mering: Rainer Hampp, 29-58.

Rieckmann, M. \& C. Schank (2016): Sozioökonomisch fundierte Bildung für nachhaltige Entwicklung. Kompetenzentwicklung und Werteorientierungen zwischen individueller Verantwortung und struktureller Transformation. In: SOCIENCE 1, 65-79.

Rieckmann, M. (2017): Bildung für nachhaltige Entwicklung in der Großen Transformation - Neue Perspektiven aus den Buen Vivir- und Postwachstumsdiskursen. In: Emde, O., U. Jakubczky, B. Kappes \& B. Overwien (Hrsg.): Mit Bildung die Welt verändern? Globales Lernen für eine nachhaltige Entwicklung. Opladen, Berlin, Toronto: Barbara Budrich (Ökologie und Erziehungswissenschaft), 147-159.

Schoof-Wetzig, D. (2014): Auswertung der Nutzung des Orientierungsrahmens zum Lernbereich Globale Entwicklung im Rahmen einer Bildung für nachhaltige Entwicklung. https://www.engagement-global.de/lernbereich-globale-entwicklung.html?file=files/2_Mediathek/ Mediathek_EG/Angebote_A_Z/Lernbereich_Globale_ Entwicklung/Baden-Wuerrtemberg/EG_Auswertung_ Nutzung_Orientierungsrahmen.pdf (25.6.2018).

Singer-Brodowski, M. (2016): Transformative Bildung durch transformatives Lernen. Zur Notwendigkeit der erziehungswissenschaftlichen Fundierung einer neuen Idee. In: Zeitschrift für internationale Bildungsforschung und Entwicklungspädagogik 1, 13-17.

Ulrich-Riedhammer, E. (2014): Die ethische Dimension von Nachhaltigkeit und Gerechtigkeit im Geographieunterricht mitdenken. In: Müller, M., I. Hemmer \& M. Trappe (Hrsg.): Nachhaltigkeit neu denken. Rio+X: Impulse für Bildung und Wissenschaft. München: oekom, 291-298.

Vanhulst, J. \& A. Beling (2014): Buen vivir: Emergent discourse within or beyond sustainable development? Methodological and Ideological Options. In: Ecological Economics 101, 54-63.

Vences, U. (2013): Buen Vivir - Vom „Guten Leben“ und dem Recht darauf. In: Hispanorama 141, 32-40.

Verband Entwicklungspolitik Niedersachsen e.V. (Hrsg., 2014): Voll konkret! Methoden zum Globalen Lernen. Das Begleitheft zum Comic „Voll global!“. http:// www.ven-nds.de/images/ven/projekte/globales_lernen/ voll\%20konkret.pdf (25.6.2018).

Verein Niedersächsischer Bildungsinitiativen e.V. (Hrsg., 2014): Die große Globalisierung für kleine Leute. Globales Lernen mit Grundschulkindern. Grundschule. http:// www.globaleslernen.de/sites/default/files/files/pages/ vnb_broschuere_webansicht_schule_0.pdf (25.6.2018).

Welthaus Bielefeld (Hrsg., 2012): „Buen Vivir - Was heißt Gutes Leben?“. Bildungsmaterial für die Fächer Praktische Philosophie und Religion (Kl. 9+10). http://www.modellschulen-globales-lernen.de/fileadmin/user_upload/ modell/Buen_Vivir/Buen_Vivir_web.pdf (25.6.2018). 